\title{
A Systematic Review of Race and Ethnicity in Hepatitis C Clinical Trial Enrollment
}

Julius Wilder, M.D., Ph.D., Anirudh Saraswathula, Vic Hasselblad, Ph.D., Andrew Muir, M.D., M.H.S.

Abstract: The African American/Black population in the United States (US) is disproportionately affected by hepatitis $\mathrm{C}$ virus (HCV) and has lower response rates to current treatments. This analysis evaluates the participation of African American/Blacks in North American and European HCV clinical trials. The data source for this analysis was the PubMed database.

Randomized controlled clinical trials (RCT) on HCV treatment with interferon $2 \mathrm{a}$ or $2 \mathrm{~b}$ between January 2000 and December 2011 were reviewed. Inclusion criteria included English language and participants 18 years or older with chronic HCV. Exclusion criteria included non-randomized trials, case reports, cohort studies, ethnic specific studies, or studies not using interferon-alfa or PEG interferon. Of the 588 trials identified, $314(53.4 \%)$ fit inclusion criteria. The main outcome was the rate of African American/ Black participation in North American HCV clinical trials. A meta-analysis comparing the expected and observed rates was performed.

Of the RCT's that met search criteria, $123(39.2 \%)$ reported race. Clinical trials in North America were more likely to report racial data than European trials. Racial reporting increased over time. There was a statistically significant difference among the expected and observed participation of African Americans in HCV clinical trials in North America based on the prevalence of this disease within the population.

The burden of HCV among African Americans in North America is not reflected in those clinical trials designed to treat HCV. Research on minority participation in clinical trials and how to increase minority participation in clinical trials is needed.

Abbreviations: HCV, hepatitis C virus; US, United States; RCT, randomized controlled trial; FDA, food and drug administration

Keywords: Hepatitis C $\mathbf{n}$ Clinical Trial $\mathbf{n}$ African American/Black

Author affiliations: Julius Wilder, Division of Gastroenterology, Duke University School of Medicine, Duke Clinical Research Institute; Anirudh Saraswathula, Trinity College of Arts and Sciences, Duke University; Vic Hasselblad, Duke Clinical Research Institute; Andrew Muir, Division of Gastroenterology, Duke University School of Medicine, Duke Clinical Research Institute

Correspondence: Julius M. Wilder, M.D., Ph.D., Duke Clinical Research Institute, P.O. Box 17969, DUMC 3850, Durham, NC 27715, tel: (919) 668-7193, fax: (919) 668-7164, email: julius. wilder@duke.edu

Copyright $\odot 2016$ by the National Medical Association

http://dx.doi.org/10.1016/j.jnma.2015.12.004

\section{INTRODUCTION}

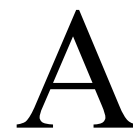

frican Americans are disproportionately affected by Hepatitis C Virus (HCV) in the United States.

$\mathrm{HCV}$ infection is a leading cause of cirrhosis, hepatocellular carcinoma and is the most common indication for liver transplantation in the United States. ${ }^{1}$ An estimated 3.2 million persons in the United States have chronic $\mathrm{HCV}$ infection and carry risk of severe morbidity and increased mortality. ${ }^{2,3}$ While African Americans comprise approximately $13 \%$ of the US population, they make up approximately $23 \%$ of Americans with hepatitis C. ${ }^{4}$ A report using NHANES III data (1999-2002) found that the rate of a positive $\mathrm{HCV}$ antibody test was higher in blacks than in whites $(3.2 \%$ versus $1.5 \%)$. Black men had higher rates of infection, and the highest prevalence rate was $9.8 \%$ among black men ages 40 to 49 years. ${ }^{5,6}$

$\mathrm{HCV}$ treatment is undergoing rapid evolution with the first generation protease inhibitors released in 2011 and now multiple direct acting antivirals regimens that re interferon free. ${ }^{7,8}$ Although the burden of HCV on African Americans is significant, this population has been traditionally underrepresented in clinical trials. Previous newmedicationshavebeenreleased withlimitedexperience in African Americans, and post-approval studies in the past have consistently demonstrated lower $\mathrm{HCV}$ treatment response rates among African Americans. ${ }^{9-11}$ The purpose of this study was to review the participation of African Americans in HCV trials during the first decade of this millennium through the development of the first generation protease inhibitors.

\section{METHODS}

\section{Literature Search}

We performed the literature search in March 2012 using the PubMed search engine (US National Library of Medicine, National Institutes of Health, Bethesda, M.D., USA). With the dates of publication limited to between 2000 and 2011, we entered the following MeSH terms/supplementary concepts into the search: "Hepatitis C, Chronic," "interferon alfa-2a," and "interferon alfa-2b." The aforementioned terms were also searched for in titles and abstracts. Only the results of this search were further processed for data collection and analysis. The collection of results was not supplemented with other outside articles.

\section{Data Collection}

The PubMed search provided 588 search results, which were later screened for inclusion (see below). The results encompassed 132 different journals and 406 unique 
authors. The research team manually collected the results using a pre-designed data collection form. Year of publication, name of first author, and journal were used to identify results in the form. In addition, several other fields were created to itemize data collected from individual results. In addition to basic information, the following data were collected: "Genotypes studied," "HIV co-infection $(\mathrm{Y} / \mathrm{N})$," "US/Canadian sites (Y/N)," "Latin American sites $(\mathrm{Y} / \mathrm{N})$," "European sites $(\mathrm{Y} / \mathrm{N})$, , "Asian sites $(\mathrm{Y} / \mathrm{N})$, "African sites (Y/N)," "Australian/NZ sites (Y/N)," "IFN alfa or PEG- IFN alfa used (Y/N)," "Ribavirin used (Y/ $\mathrm{N})$," "DAA used (Y/N)," "Amantadine used(Y/N)," "Other medications used," "Race reported $(\mathrm{Y} / \mathrm{N})$," "Total patients," "Number white," "Number black," "Number Latino," "Number Asian," and "Number other". Studies were designated as "reporting race" or "reporting ethnicity" if they included a statement or table explaining patient racial or ethnic makeup.

Inclusion criteria were English articles with participants aged older than 18 years with chronic HCV. Exclusion criteria were non-human studies, non-randomized trials, letters/case reports, cohort studies, articles not reporting outcomes of interest or primary data (editorials, reviews, secondary analyses), studies enrolling specific ethnic groups, studies specifically enrolling patients with significant secondary conditions (e.g. thalassemia, cryoglobulinemia, coagulation disorders, etc.), or studies not using interferon-alfa or PEG-interferon.

\section{Definitions and Statistical Analysis}

Given that multicenter studies were conducted in multiple countries and regions of the world, trial site designations were regional descriptions designated with the terms "partially" or "only". The regions of interest in this analysis were North America and Europe. "Partially" indicated that a particular region was at least one of the trial sites, while "only" indicates that there were no other regions among the trial sites. "Partially" data groups were much larger than "only" groups, and were also skewed towards the mean reporting statistics.

We obtained HCV prevalence data from Armstrong, et al., a large survey by the National Center for Health Statistics reporting HCV prevalence in the United States between 1999 and 2002. ${ }^{4}$ We hypothesized that the expected rate of African Americans in the HCV therapy clinical trials should be equal to the relative burden of this disease among African Americans within the HCV population. Using the data from Armstrong, et al., we estimated that African Americans make up approximately 23\% of people in the United States with hepatitis C. For this analysis, we selected the HCV trials that included only North American or Canadian subjects.

The proportions of African-American subjects in studies of hepatitis $\mathrm{C}$ were summarized using a random effects model because of the clear heterogeneity of the studies. ${ }^{12}$ We used the method of DerSimonian and Laird ${ }^{13}$ as implemented in Comprehensive Meta-Analysis. ${ }^{14}$ The estimated proportion was 0.148 with 95 percent confidence limits of 0.126 to 0.174 . As a sensitivity analysis we calculated estimates using two other models as implemented in the FAST*PRO software. ${ }^{15,16}$ The estimate for the empirical Bayes model was 0.145 (95 percent confidence interval of 0.118 to 0.175 ) and the estimate for the beta-binomial model was 0.158 (95 percent confidence interval of 0.130 to 0.188 ). All models clearly exclude the reported U.S. rate of 0.23 .

\section{RESULTS}

Using the systematic review, 588 clinical trials on hepatitis $\mathrm{C}$ were originally identified. Of the 588 trials initially identified, 314 (53.4\%) were randomized controlled trials (Figure 1). The trials identified within this analysis were representative of clinical trials from both North America as well Europe conducted between 2000 and 2011. Of the 314 eligible trials, $69(22.0 \%)$ took place only within North American and 143 (45.5\%) took place only within Europe (Figure 1). Many trials were multinational studies and therefore included North America, Europe, and other regions of the world. We identified 113 $(36.0 \%)$ multinational trials that included North America and 187 (59.6\%) multinational trials that included Europe (Figure 1).

Figure 1. Results of PubMed literature search.
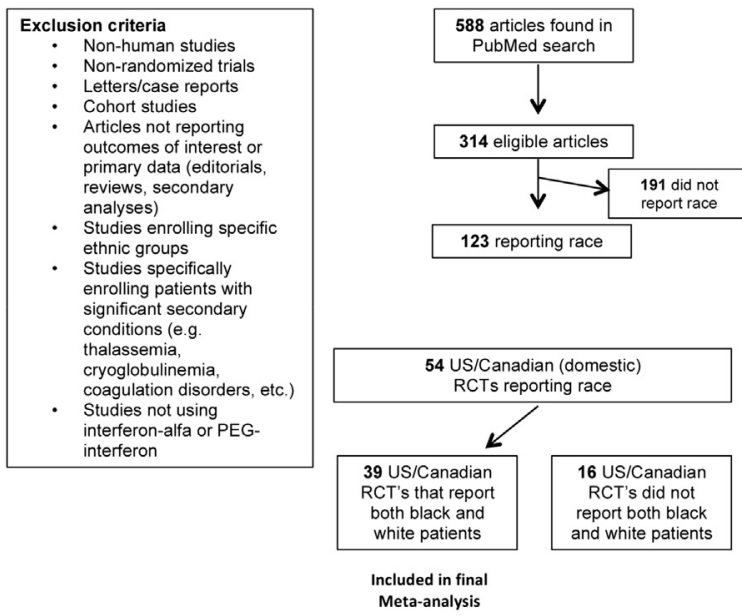


\section{Reporting of Racial Data}

Many of the trials that met search criteria did not include race or ethnicity when reporting results. Data on ethnicity was collected and reported less then racial data. Furthermore, when ethnicity was included, clear definitions for ethnicity were not apparent. Often, ethnicity was inappropriately used interchangeably with race. The data on ethnicity were also often incomplete for respective datasets ( $\mathrm{N}$ not equal to 100). The lack of data on ethnicity combined with the inaccurate ethnic data that were reported made statistical analyses of the ethnicity of the clinical trials problematic.

Of the 314 RCT's that met search criteria, 123 (39.2\%) reported race (Figure 1). The likelihood of race being reported at all within an RCT appeared to be impacted by where the RCT was conducted. North American trials reported race at a higher rate than European trials. Among trials conducted only within North America, 78.3\% (54/ 69) reported race. Conversely, only $14.7 \%$ (21/143) of those trials conducted solely within Europe reported race (Figure 2). A similar pattern was seen among multinational trials where North American multinational trials reported race in the results more frequently than European multinational trials.

The percentage of trials reporting race prior to and after 2005 were calculated to evaluate a potential change in racial reporting over time. There appeared to be an increase in racial reporting among all clinical trials over time. Racial reporting among clinical trials that only

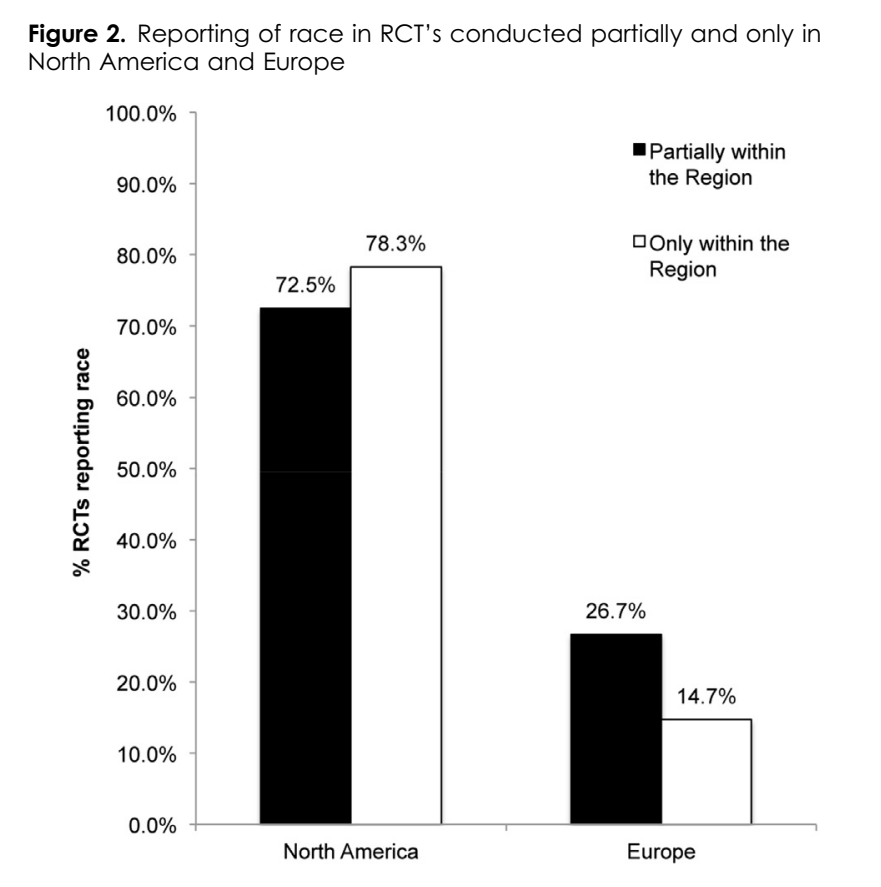

occurred within North America was essentially stable at $76.9 \%(20 / 26)$ before 2005 and 79.1\% (34/43) after 2005 . A significant increase occurred among those RCT's conducted solely in Europe. While the percentage of European studies reporting race prior to 2005 was $3.2 \%(2 / 62)$, this number increased to $23.5 \%(19 / 81)$ after $2005\left(\chi^{2}(1)=\right.$ $12.52, p<.001)$. A similar pattern was seen among the multinational trials within this analysis as well.

\section{African American Participation within Hepatitis C Clinical Trials}

While African Americans make up approximately 23\% of those within the US with $\mathrm{HCV}^{4}$ they are underrepresented with respect to their participation within clinical trials. Of those clinical trials conducted within the US only, $13.1 \%$ $(2994 / 22,832)$ of study participants were African American (Figure 3). The percentage of white study participants among RCT's conducted solely within the US was $75.3 \%$ (17201/22832) (Figure 3). Over time, there has been no change in African American participation within hepatitis $\mathrm{C}$ clinical trials conducted within the US. The percentage of African Americans among RCT participants in the US was $13.1 \%$ before and after 2005 . The percentage of white participants in hepatitis C RCTs was also essentially unchanged with a minimal decrease from $76.4 \%$ prior to 2005 to $75.2 \%$ after 2005 .

A meta-analysis was performed to examine the difference in the observed and expected percentage of African Americans in North American clinical trials. Within this

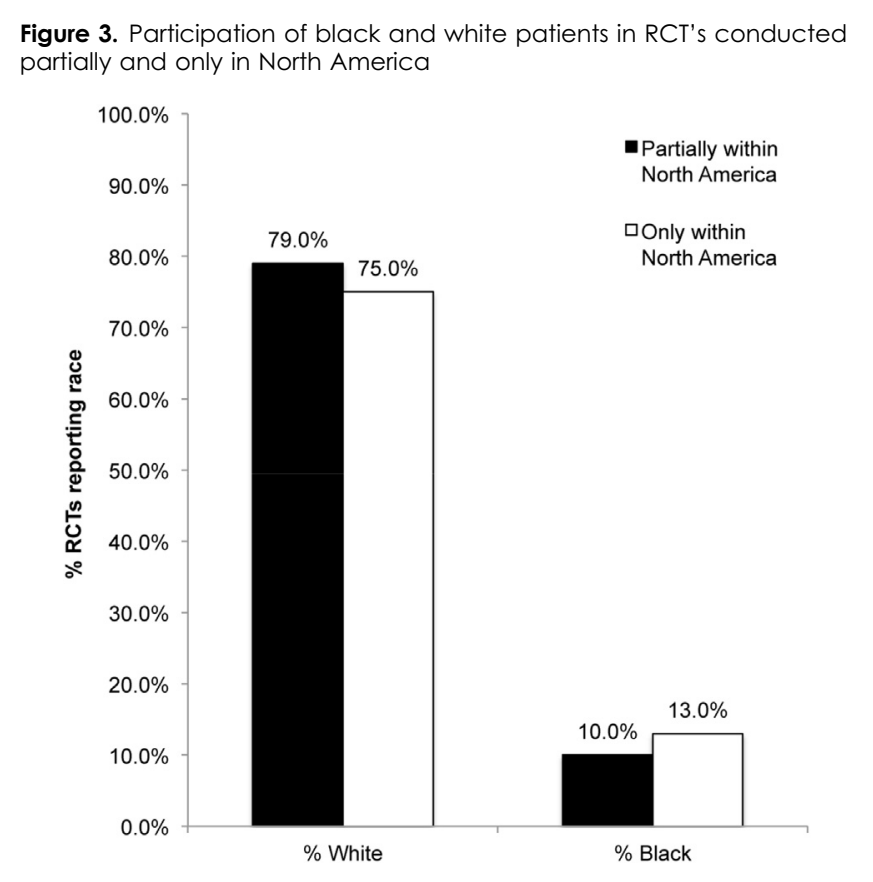


Figure 4. Rate of African American/Black Participation in North American Hepatitis C Clinical Trials from 2000-2011

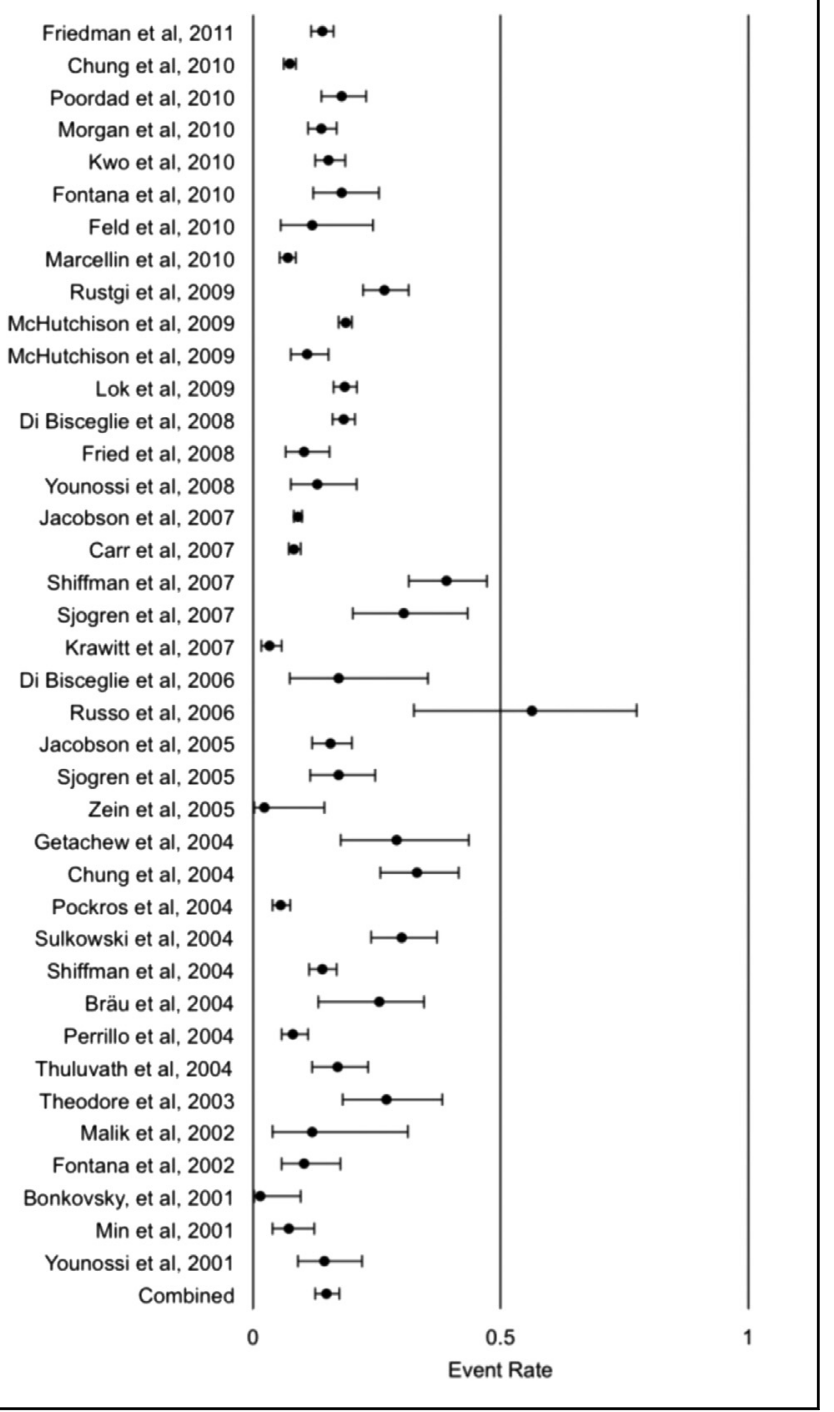

analysis there were 22,832 subjects, of which 2,994 were African American. The observed rate for the meta-analysis was 0.148 (95\% CI, 0.126 to 0.174$)$. This meta-analysis of African American participation among North American clinical trials showed that the difference between the observed and expected percentage of African Americans within hepatitis $\mathrm{C}$ clinical trials was statistically significant $(p<.0001$; Figure 4).

\section{DISCUSSION}

The goal of this analysis was to evaluate the participation of African Americans/Blacks within HCV clinical trials. Of the 314 RCTs that met search criteria, only 123 (39.2\%) reported race. The reasons for such low reporting of racial and ethnic data on study participants are unclear. One potential explanation may be related to the lack of consensus on how to collect and report these often selfreported racial and ethnic variables. The differences between racial reporting within the US and Europe are likely related to increased emphasis on racial disparities, specifically among African Americans, within the US. The reason for the increase in reporting of racial outcomes in Europe after 2005 is unclear. Interestingly, the FDA approved in June 2005 a combination medication of isosorbide dinitrate and hydralazine hydrochloride named BiDil $\AA$, which was the first drug approved by the agency to treat a disease in patients identified by race (heart failure in African Americans). ${ }^{17}$ Furthermore, that same year in September 2005 the FDA published a document entitled "Guidance for Industry: Collection of Race and Ethnicity Data in Clinical Trials." " This document made recommendations concerning the use of Office of Management and Budget (OMB) designations for collecting of racial and ethnic data in clinical trials.

Given the surprisingly low percentage of eligible RCTs that reported race at all, a standardized method of collecting and reporting racial and ethnic data should be considered. A standardized approach would help ensure consistent and accurate collection of this important information. While the US food and drug administration (FDA) has published recommendations on the collection of racial and ethnic data, this information is used as a guide and not considered a requirement. There is no formal requirement that racial and ethnic data be collected in clinical trials. By standardizing this as a requirement, appropriate collection of racial and ethnic data and correct reporting of this data would be ensured. Furthermore, areas where there is a lack of diversity with respect to clinical research would be more easily identified and appropriate measures could then be taken to address the disparity.

This analysis demonstrated that the burden of $\mathrm{HCV}$ within the African American/Black populations within the United States is not reflected in the diversity of clinical trial participants. The percentage of African Americans observed participating in hepatitis $\mathrm{C}$ clinical trials was approximately half the expected amount. Hence, the group of people most significantly affected by this disease is underrepresented in clinical trials created to treat the disease.

Identification of viable treatment options within any population for any disease is dependent on inclusion of that population in the research. Intrinsic factors (genetic, metabolic) and external factors (social, economic, cultural) can potentially impact treatment outcomes., $90,10,19-22$ Diverse populations within clinical trials help identify safe and efficacious treatment regimens for diverse 
populations of men and women. One major barrier to minority participation in clinical research trials is recruitment. Minority populations must be engaged to participate in research in a way that helps alleviate concerns related to trust $^{23}$ and those conducting the research must commit to pursuing increased diversity within clinical trials. A lack of minority participation within clinical trials may lead to increased health disparities, even in the setting of new and advanced therapies for treatment of a disease.

A major limitation in our analysis of minority participation in hepatitis $\mathrm{C}$ clinical trials was the lack of reporting of racial and ethnic data in the literature. As discussed earlier, it is unknown if this is reflective of a lack of collecting or reporting racial data. Nonetheless, this greatly limited our ability to analyze minority participations within clinical trials. The actual percentage of African Americans/Blacks within the included RCTs may be higher, but due to a lack of reporting of this data, we may be underestimating the population totals.

The comparison of US and European trials is limited by the search criteria and database used for this analysis. African American is a designation not used consistently in Europe. We did use the search term Black, but this term may reflect different populations within the US versus Europe. The database used for identification of clinical trials was PubMed. This clinical research database has a US bias and may not accurately reflect all European trials that met criteria. Finally, there does exist significant heterogeneity among the African American population within the United States and Canada, which is reflected in the Q-value of 670.4. for 38 degrees of freedom $(p<0.001)$. However, this heterogeneity was expected and taken into consideration, because the distribution of African Americans across the United States is far from uniform.

In conclusion, the participation of minorities within hepatitis C clinical trials between 2000 and 2011 in the US and Europe was not consistently reported. Overall, US trials reported race more consistently than European trials, and reporting of race increased after 2005. African Americans were underrepresented within these clinical trials given the burden of Hepatitis $\mathrm{C}$ within this population. The importance of race as an internal (genetic, metabolic) and external (social, economic, cultural) factor in disease outcomes is well known, and standardization of collecting and reporting racial/ethnic data may ensure consistent and accurate reporting of racial/ethnic data from clinical trials. Research is needed to better understand reasons for lower enrollment of racially and ethnically diverse populations, and to create ways to improve participation of racially and ethnically diverse populations in clinical trials.

\section{DISCLOSURES}

While no funding was directed to obtaining the supply of data, below are all financial disclosures and acknowledgements.

1. Julius Wilder was supported by the Duke NIDDK Grant No. 5T32-DK7568-22.

2. Anirudh Saraswathula was supported by the Boebel Family Fund as well as the Duke Undergraduate Internship Funding Program award.

3. Andrew Muir has received research funding and served as a consultant for: Abbvie, Achillion, BMS, Gilead, GSK, Merck, and Vertex. He also received research funding from Roche. Funders had no role in the systematic review.

4. Vic Hasselblad has no disclosures.

\section{REFERENCES}

1. Shepard, C. W., Finelli, L., \& Alter, M. J. (Sep 2005). Global epidemiology of hepatitis $C$ virus infection. The Lancet infectious diseases, 5(9), 558-567.

2. Lavanchy, D. (Feb 2009). The global burden of hepatitis C. Liver international: official journal of the International Association for the Study of the Liver, 29(Suppl 1), 74-81.

3. Centers for Disease Control and Prevention. (2008). Hepatitis C Information for Health Professionals. Centers for Disease Control and Prevention. http://www.cdc.gov/hepatitis/ hcv/.

4. Armstrong, G. L., Wasley, A., Simard, E. P., McQuillan, G. M., Kuhnert, W. L., \& Alter, M. J. (Jun 16 2006). The prevalence of hepatitis C virus infection in the United States, 1999 through 2002. Annals of internal medicine, 144(10), 705-714.

5. NIH consensus statement on management of hepatitis C: 2002 . Consensus Development Conference presented at $\mathrm{NIH}$ consensus and state-of-the-science statements; Jul, 2002.

6. Alter, M. J., Kruszon-Moran, D., Nainan, O. V., et al. (Aug 19, 1999). The prevalence of hepatitis $C$ virus infection in the United States, 1988 through 1994. The New England journal of medicine, $347(8), 556-562$.

7. Jacobson, I. M., McHutchison, J. G., Dusheiko, G., et al. (Jul 23 $2011)$. Telaprevir for previously untreated chronic hepatitis C virus infection. The New England journal of medicine, 364 (25), 2405-2416.

8. Poordad, F., McCone, J., Bacon, B. R., et al. (Apr 31 2011). Boceprevir for untreated chronic HCV genotype 1 infection. The New England journal of medicine, 364 (13), 1195-1206.

9. McHutchison, J. G., Poynard, T., Pianko, S., et al. (Nov 2000). The impact of interferon plus ribavirin on response to therapy in black patients with chronic hepatitis $\mathrm{C}$. The International 
Hepatitis Interventional Therapy Group. Gastroenterology, $119(5), 1317-1323$.

10. Muir, A. J., Bornstein, J. D., Killenberg, P. G., \&, Group ACHT (Jun 27 2004). Peginterferon alfa-2b and ribavirin for the treatment of chronic hepatitis $C$ in blacks and non-Hispanic whites. The New England journal of medicine, 350(22), 2265-2271.

11. Conjeevaram, H. S., Fried, M. W., Jeffers, L. J., et al. (Aug 2006). Peginterferon and ribavirin treatment in African American and Caucasian American patients with hepatitis C genotype 1. Gastroenterology, 131(2), 470-477.

12. Skellam, J. G. (1948). A probability distribution derived from the binomial distribution by regarding the probability of success as variable between the sets of trials. Journal of the Royal Statistical Society Series $B$.

13. DerSimonian, R., \& Laird, N. (Sep 1986). Meta-analysis in clinical trials. Controlled clinical trials, 7(3), 177-188.

14. SAS Institute I. (1999). SAS/STAT® User's Guide. SAS Institute, Inc.

15. Eddy, D. M., \& Hasselblad, V. (1992). Fast Pro. Academic Press.

16. Hedges, L. V., \& Olkin, I. (1985). Statistical methods for metaanalysis. Orlando: Academic Press.

17. Yancy, C. W., Fowler, M. B., \& Colucci, W. S. (2001). Race and the response to adrenergic blockade with carvedilol in patients with chronic heart failure. The New England journal of medicine.
18. US Dept. of Health and Human Services, Food and Drug Administration, Center for Drug Evaluation and Research, Center for Biologics Evaluation and Research, Center for Devices and Radiologic Health. (Sep 2005). Collection of Race and Ethnicity Data in Clinical Trials. Rockville, M.D.: Food and Drug Administration.

19. Brown, R., Jacobson, I., Afdhal, N., et al. (2002). Racial/ethnic differences in hematologic toxicity of pegylated interferon and ribavirin therapy: analysis of the WIN-R trial. Hepatology (Baltimore, Md.).

20. Jeffers, L. J., Cassidy, W., Howell, C. D., Hu, S., \& Reddy, K. R. (Ju) 2004). Peginterferon alfa-2a (40 kd) and ribavirin for black American patients with chronic HCV genotype 1. Hepatology (Baltimore, Md.), 39(6), 1702-1708.

21. Reddy, K. R., Hoofnagle, J. H., Tong, M. J., et al. (Sep 1999). Racial differences in responses to therapy with interferon in chronic hepatitis C. Consensus Interferon Study Group. Hepatology (Baltimore, Md.), 30(3), 787-793.

22. Wiley, T. E., Brown, J., \& Chan, J. (Apr 2002). Hepatitis C infection in African Americans: its natural history and histological progression. The American journal of gastroenterology, 97(3), 700-706.

23. Rusert, B. (2009). "A Study in Nature": The Tuskegee Experiments and the New South Plantation. Journal of Medical Humanities. 